\title{
Australia set to test insulin 'vaccine' for juvenile diabetes
}

A 'vaccine-like' approach to juvenile diabetes, set to be tested in a clinical trial in Australia, aims to test whether delivering insulin through the nose can keep the immune system from attacking the body's own cells.

Vaccines prime the immune system to attack invaders and protect the body. But in type 1 diabetes, it is the T cells that attack and destroy insulin-secreting beta cells in the pancreas.

In a phase 2 trial announced in December, Australian researchers are testing whether nasal insulin delivered to people at risk of type 1 diabetes can prevent the disease by training their immune systems to tolerate beta cells.

Edwin Gale, head of the diabetes research unit at the University of Bristol, says although it is too early to speculate on whether the approach can prevent type 1 diabetes, it could add to scientists' understanding of immune tolerance.

Calling the approach 'vaccine-like' has provoked some skepticism from experts who say vaccinating against an autoimmune disease is inherently contradictory.

Warwick Anderson, chief executive of Australia's National Health and Medical
Research Council, which co-funded the trial, says the term is a bit of a misnomer. "That word 'vaccine' stuck early on, 'immune regulation' would be better."

Insulin administered via the nose or mouth stimulates the immune system via the mucosal lining but is not absorbed further and does not affect blood glucose levels. A phase 1 trial completed in 2004 found immune responses similar to those seen in mouse studies.

A significant increase in antibodies to insulin accompanied a decrease in $\mathrm{T}$-cell response, the first demonstration of this effect in humans. Project leader Len Harrison, of the Walter and Eliza Hall Institute in Melbourne, says the approach is based on the phenomenon of mucosal tolerance first reported in the 1940s.

"It seems paradoxical because insulin antibodies are a marker for the disease, but there is no evidence that antibodies themselves cause damage to beta cells," he says. The more important thing, he adds, is that the method suppresses the immune response to insulin.

In a separate project, Matthias von Herrath is investigating whether those who have recently contracted the disease would benefit from a combination of nasal insulin and drugs to suppress the immune system.

"Our studies have shown that such combotherapies are much more effective," says von Herrath, head of the Immune Regulation Lab at the La Jolla Institute for Allergy and Immunology.

Previous attempts to show mucosal tolerance in people with type 1 diabetes have all failed (Diabetes Care 27, 2348-2355; 2004). But those studies involved individuals with end-stage disease who received oral insulin.

Delivering insulin through the nose would prevent it from degrading before it reaches the mucosa, Harrison says. The phase 1 trial suggested that although the treatment does not eliminate the $\mathrm{T}$ cells that destroy beta cells, it might enhance the $\mathrm{T}$ cells that help make antibodies. "You would have a mixture, it's a balance between the good guys and the bad guys," Harrison says.

At least 13,200 relatives of people with type 1 diabetes will be screened in order to identify 264 participants at high risk. Results are expected in seven years.

Simon Grose, Canberra

\section{US proposal to expand access to untested drugs draws fire}

Should those who are sick or dying and have exhausted all other options be able to try drugs that haven't yet been approved? The US Food and Drug Administration (FDA) seems to think so and in December proposed a controversial scheme to help people get the drugs they need.

The move could cause chaos, jeopardizing drug development and exposing scores of people to unsafe drugs, experts caution.

The proposal is controversial not least because it allows people to try drugs in early stages of testing - in rare circumstances, even those that have never been studied in humans. Some of these might later prove to cause nasty side effects or even death, critics note.

The most common way for an individual to get an experimental drug is to enroll in a clinical trial. Not everyone qualifies for these studies, however, and some participants receive a placebo rather than the drug.

The FDA has for decades allowed people dying of certain "life-threatening" diseases, such as AIDS and cancer, to try experimental drugs outside of clinical trials. But the process of applying on the grounds of 'compassionate use' is convoluted, sometimes requiring inside contacts at a company or at the FDA.

For example, the rules also allowed those

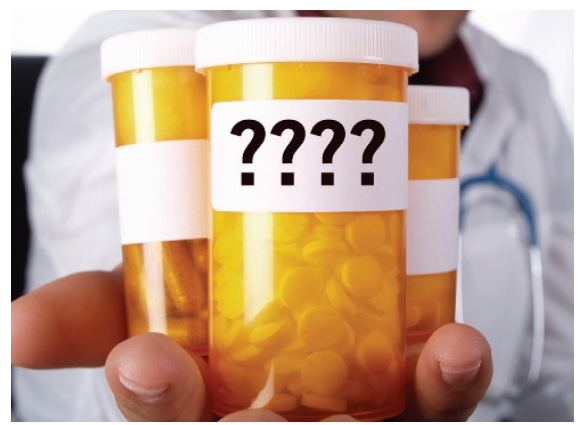

Hidden dangers: New rules would allow those with serious illnesses to take unapproved drugs.

with a "serious" illness, such as depression and rheumatoid arthritis, to apply for experimental drugs, but the definition of serious was buried in a separate set of regulations.

The new rules clarify those ambiguities, spelling out who is eligible for these drugs, how to request them and how much companies can charge for them. The proposal is under a 90-day review that ends in March.

"We believe these rules, when finalized, will help reach out to other populations," says Rachel Behrman, deputy director of the FDA's office of medical policy. "We will review every request."

As people learn more about these rules, however, the agency might find itself flooded with requests for investigational drugs for anything from depression to cancer. Drug makers, which have traditionally resisted supplying experimental drugs because of liability issues, might also succumb to the demand. The proposed rules might in part be a response to a lawsuit brought by the Virginia-based advocacy group Abigail Alliance (Nat. Med. 12, 596, 2006).

"Making this a little more accessible may cause the well-informed patients to be more demanding," says Richard Kingham, a partner at the law firm Covington and Burling. "And history shows that if there's pressure, a company will consider supplying the drug."

Most antidepressants work for only about half of those who take them. If companies release those drugs, it could paint a misleading picture of the drug's safety and efficacy. It could also take away incentives for individuals to enroll in clinical trials.

Expanding access to experimental drugs "could be a disaster for the drug approval process," says Jerry Menikoff, a health law expert at the University of Kansas. "The only reason some people enroll in studies is because they need the drug."

Emily Waltz, New York 